\title{
BMJ Open Effect of free distribution of medicines on the process of care for adult patients with type 1 and type 2 diabetes and hypertension: post hoc analysis of randomised controlled trial findings
}

\author{
Onella Charles, ${ }^{1,2}$ Hannah Woods, ${ }^{2}$ Muhamad Ally, ${ }^{2}$ Braden Manns, ${ }^{3,4,5,6}$ \\ Baiju R Shah, ${ }^{7,8,9,10}$ Ri Wang, ${ }^{2}$ Nav Persaud (1) ${ }^{2,8,9,11}$
}

To cite: Charles 0 , Woods $\mathrm{H}$ Ally $\mathrm{M}$, et al. Effect of free distribution of medicines on the process of care for adult patients with type 1 and type 2 diabetes and hypertension: post hoc analysis of randomised controlled trial findings. BMJ Open 2021;11:e042046. doi:10.1136/ bmjopen-2020-042046

- Prepublication history for this paper is available online To view these files, please visit the journal online (http://dx.doi. org/10.1136/bmjopen-2020042046).

Received 23 June 2020 Revised 02 February 2021 Accepted 21 February 2021

Check for updates

(C) Author(s) (or their employer(s)) 2021. Re-use permitted under CC BY-NC. No commercial re-use. See rights and permissions. Published by BMJ.

For numbered affiliations see end of article.

Correspondence to

Dr Nav Persaud;

nav.persaud@utoronto.ca

\section{ABSTRACT}

Objectives The Carefully Selected and Easily Accessible at No charge Medicines randomised controlled trial showed that patients receiving free access to medicines had improved diabetes and hypertension outcomes compared with patients who had usual access to medicines. In this study, we aimed to test the impact of providing free access to medicine to people with diabetes and hypertension on process of care indicators.

Design In this post hoc analysis of randomised controlled trial findings, we identified process of care indicators for the management of diabetes and hypertension using relevant guidelines. The following process of care indicators were identified for diabetes management: encounters with healthcare professionals, blood pressure measurements, self-monitoring of blood glucose, annual eye and foot examination, annual administration of the influenza vaccine, and laboratory testing for glycated haemoglobin (HbA1c), low-density lipoprotein-cholesterol, serum creatinine and urine albumin to creatinine ratio. We identified the following process of care indicators for hypertension: encounters with healthcare professionals, blood pressure measurements, self-measuring of blood pressure, and serum tests for electrolytes, $\mathrm{HbA1c}$, lipids and creatinine. Chart extractions were performed for all patients and the indicators for diabetes and hypertension were recorded. We compared the indicators for patients in each arm of the trial.

Results The study included 268 primary care patients. Free distribution of medicines may improve self-monitoring behaviours (adjusted rate ratio (aRR) 1.30; 95\% Cl 0.66 to 2.57) and reduce missed primary care appointments for patients with diabetes (aRR 0.80; 95\% $\mathrm{Cl} 0.48$ to 1.33) or hypertension (aRR $0.41 ; 95 \% \mathrm{Cl} 0.18$ to 0.90 ). Free distribution may also reduce primary care and consultant appointments and laboratory testing in patients with hypertension.

Conclusions Improving medicine accessibility for patients with diabetes and hypertension not only improves surrogate health outcomes but also improves the patient experience and may also reduce healthcare costs by encouraging self-monitoring.
Strengths and limitations of this study

- The study is based on a randomised controlled trial.

- Despite the fact that this was a post hoc analysis and randomisation was not stratified based on these characteristics, we found that the groups were largely balanced.

- Associations identified during post hoc analyses could be spurious and thus the findings should be viewed as hypothesis-generating.

- The trial this study was based on was conducted with primary care patients in a high-income country who reported cost-related non-adherence, and the findings may not apply in other settings.

Trial registration number The randomised controlled trial mentioned is clinicaltrials.gov identifier: NCT02744963.

\section{INTRODUCTION}

Managing chronic diseases such as diabetes and hypertension with effective medicines and healthcare services can save lives and reduce complications, yet many people do not receive guideline-recommended care. ${ }^{1-3}$ One important barrier to optimal care is costrelated non-adherence which was reported by $9.6 \%$ of people who had received a prescription in the past year. ${ }^{4}$ Cost-related nonadherence could undermine the provision of healthcare services as people may avoid participating in care if they cannot afford prescribed medicines. ${ }^{45}$

Many strategies have been tested to improve the process of care for chronic diseases, with varying success. Resource-intensive interventions such as financial incentives to providers and multidisciplinary changes to the primary care team are associated with modest improvements in diabetes and hypertension management. $^{6}$ Caring for patients with 
chronic diseases is expensive. ${ }^{8}$ The cost and effectiveness of interventions to improve guideline-recommended care are important to consider, since increasing access to effective treatments may reduce costs related to complications, but may increase per-patient costs related to clinicians' monitoring of treatments and more expensive health technologies. ${ }^{9}$

We recently completed the Carefully Selected and Easily Accessible at No charge Medicines (CLEAN Meds) trial, a randomised controlled trial in which patients with selfreported cost-related medication non-adherence were randomly assigned to receive free distribution of medicines from a comprehensive list of essential medicines. ${ }^{10}$ The CLEAN Meds trial found that providing Canadian primary care patients with medicines at no charge improved adherence to medication and, for patients with diabetes and hypertension, chronic disease management was improved based on some surrogate outcomes. ${ }^{10}$ As previously reported, with free distribution of medicines, glycated haemoglobin (HbAlc) levels were $0.4 \%$ lower (95\% CI $-0.76 \%$ to $0.0 \%$ ) compared with usual access, and systolic blood pressure was $7 \mathrm{~mm} \mathrm{Hg}$ lower (95\% CI -11.7 to -2.8 ) compared with usual access.

We undertook this post hoc analysis both to help understand why the intervention was beneficial in some circumstances and why the intervention did not have a large benefit in general or any benefit for some participants. Improving access to medicines could improve the process of care as patients who are non-adherent may lack motivation for participating in care. Participation in diabetes education is associated with both better quality of diabetes care and greater adherence to diabetes medicines, indicating that medicine adherence and quality of care may improve together. ${ }^{11}$ On the other hand, improved adherence and better disease control could also lead to less participation in care. Patient-centred medical homes is associated with improved quality of diabetes care but not with better medicine adherence, suggesting that the process of care and medicine adherence can be uncoupled. ${ }^{12}$ Given the importance of medication-related adherence in patients with chronic diseases, in this post hoc analysis, we tested the impact on diabetes and hypertension process of care indicators of providing free access to medicine to people with diabetes and hypertension.

\section{METHODS \\ Patients}

We identified patients in the CLEAN Meds trial with diabetes (with or without hypertension) or only hypertension by identifying all participants prescribed at least one diabetic or antihypertensive agent at the start of the trial. Randomisation was not stratified based on these conditions. Because antihypertensives such as ACE inhibitors and angiotensin receptor blockers are a standard part of diabetes management (even when blood pressure is 'normal'), we included patients who were prescribed both a diabetic agent and an antihypertensive agent only in the diabetes group.

\section{Process of care indicators}

Using the care goals of diabetes and the Diabetes Canada Guidelines, ${ }^{13}$ we identified the following process of care indicators for the management of diabetes: encounters with healthcare professionals (in-clinic appointments and telephone appointments with primary care physicians or nurse practitioners), blood pressure measurements, self-monitoring of blood glucose (SMBG), annual eye examination (with an optometrist or ophthalmologist), foot screening examinations (foot care and/or neuropathy screening), annual administration of the influenza vaccine, and laboratory testing for HbAlc, low-density lipoprotein-cholesterol (LDL-c), serum creatinine $(\mathrm{Cr})$ and urine albumin to creatinine ratio (ACR) ${ }^{13} \mathrm{HbAlc}$ and SMBG can be used as indicators for the management of glycaemic control.

Using the guidelines and the goals of care for hypertension and the Hypertension Canada Guidelines, ${ }^{14}$ we identified the following process of care indicators for the management of hypertension: encounters with healthcare professionals, blood pressure measurements, selfmeasuring of blood pressure (at home or at the pharmacy), and serum tests for electrolytes, HbAlc, lipids and Cr.

Since a number of the recommended clinical manoeuvres and other aspects of care (eg, medicine adjustments) involve patients interacting with healthcare providers, we also assess healthcare encounters that included in-person visits and telephone encounters with primary care physicians or nurse practitioners where diabetes or hypertension was documented as being discussed.

\section{Data collection}

Patients' primary care electronic medical records (EMRs) were accessed using the PS Suite software (Telus Health) and information for the identified process of care indicators for diabetes and hypertension was identified and abstracted. Two abstracters (OC, HW) were blinded to the patients' intervention status at the time of chart abstraction. To ensure reliability of chart abstraction, each abstracter completed five chart abstractions independently and compared findings; there were no disagreements. OC, HW and MA then completed the chart abstractions for all participants.

For all patients with diabetes, starting from the patient's start date in the trial to 1 year later, the following information was recorded from each chart: number of encounters with primary care physicians and nurse practitioners related to diabetes (in-person visits and phone calls were included), number of missed primary care appointments (this is tracked and missed appointments are explicitly stated in the EMR), number of consultant (specialist physician) encounters related to diabetes, number of blood pressure measurements performed at healthcare visits, number of serum HbAlc measurements, number of serum LDL-c measurements, if serum Cr was measured 
(binary; done during the year or not), if urine ACR was measured (binary), if the patient self-monitored their blood glucose levels (binary), if an annual eye screening examination was performed (binary), if an annual foot screening examination was performed (binary) and if the annual influenza vaccine was administered (binary). We also recorded the number of new diabetes medicines each patient with diabetes was prescribed and the number of diabetes medicines they stopped taking, during the 1-year study period. All of this information was found in the charts as expected, however, influenza vaccines may have been given elsewhere, such as at a pharmacy, and may not have been fully captured in chart review.

For all patients with hypertension, starting from the patient's start date in the trial to 1 year later, the following information was recorded: total number of encounters with primary care physicians and nurse practitioners, number of consultant appointments related to hypertension, number of missed primary care appointments, number of blood pressure measurements performed at healthcare visits, number of serum electrolyte tests (any number of the following tests were included: $\mathrm{Na}, \mathrm{K}, \mathrm{Cl}, \mathrm{HCO} 3-$ and if a patient had NA, $\mathrm{K}$ and $\mathrm{Cl}$ done on the same day, this was counted as one electrolyte test), number of serum HbA1c measurements, number of serum lipid measurements (any number of the following tests were included: LDL-c, high-density lipoprotein-cholesterol (HDL-c), non-HDL-c, triglycerides, cholesterol), number of serum Cr measurements, if the patient self-measures their blood pressure either at home or at a community pharmacy (binary), and the number of new hypertension medicines each patient was prescribed and stopped taking. This information was found in the charts as expected.

\section{Data analysis}

For clinical manoeuvres that are recommended to be performed multiple times during 1 year (eg, blood pressure measurements) and for encounters with healthcare professionals, we report the rate ratios (RRs) with 95\% CIs that were estimated using a negative binomial regression model. We report unadjusted RRs and RRs adjusted for age, sex and clinic location (urban vs rural). We compared the proportion of patients in each arm receiving clinical manoeuvres that are recommended to be done only once during a 1-year period (eg, annual eye examination for people with diabetes) and report the OR with 95\% CIs that was estimated using a logistic regression model. We report unadjusted ORs and ORs adjusted for age, sex and location (urban vs rural). No p value threshold was set for these post hoc and hypothesis-generating analyses.

We also compared the net change in medications for patients with hypertension and diabetes in the intervention and control arms. As part of the intervention, some patients had to switch medicines within a class. We thus used net changes as a measure that would treat both groups similarly and captured whether or not management had 'intensified' by adding more agents.

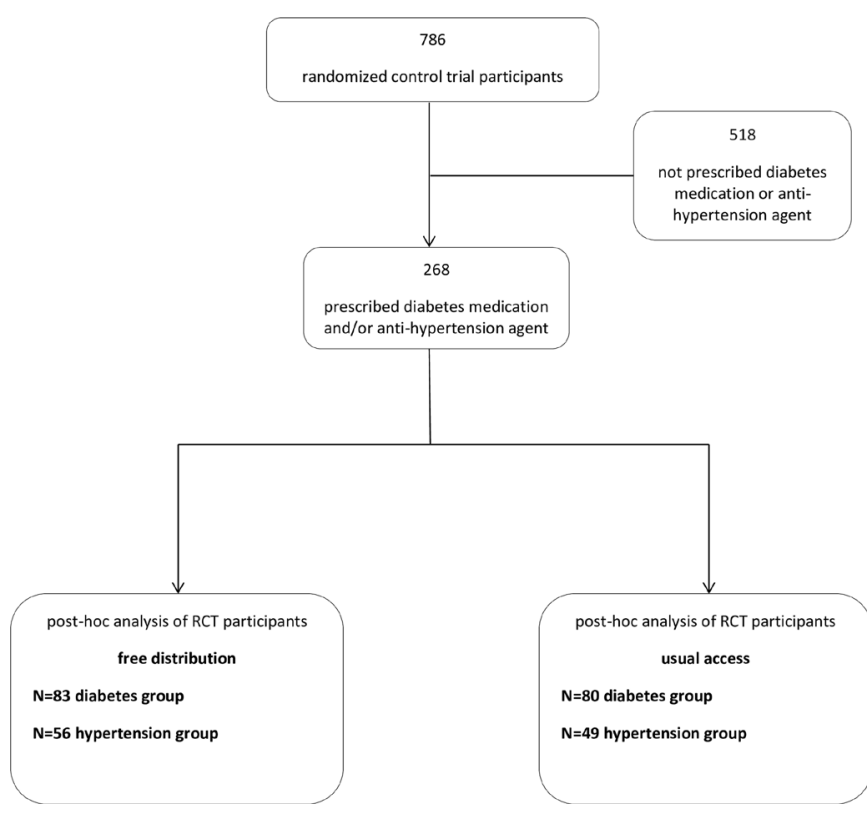

Figure 1 Flowchart illustrating study participant inclusion. $\mathrm{RCT}$, randomised controlled trial.

\section{Patient and public involvement}

Patients or the public were not involved in the design, conduct, or reporting, or dissemination plans of our research.

\section{RESULTS}

\section{Baseline characteristics}

Of the 786 patients enrolled in the CLEAN Meds trial, 163 patients were prescribed one or more medicines for diabetes and were included in the diabetes group (including 114 who were also prescribed one or more antihypertensive agents), and 105 patients were nondiabetic and prescribed one or more antihypertensive agents and included in the hypertension group. We thus included 268 participants in this study. Of the 163 patients with diabetes, 83 patients were in the intervention group receiving free distribution of medicines, while the remaining 80 patients were in the control group receiving standard access to medicines. Of the 105 patients with hypertension, 56 patients were in the intervention group receiving free distribution of medicines, and 49 patients were in the control group receiving standard access to medicines. Study participant inclusion is illustrated in figure 1.

For this post hoc analysis, the groups are balanced with the exception of hypertension in urban and rural groups. The characteristics of participants in the diabetes and hypertension groups are summarised in table 1 .

Impact of free distribution of medicines in subgroup of people with diabetes

For patients with diabetes, there were small increases in rates of serum Cr measurement (adjusted OR (aOR) 1.33; 95\% CI 0.61 to $2.91 ; \mathrm{p}=0.48$ ) but not HbAlc measurements (adjusted RR (aRR) 1.09; 95\% CI; 0.88 to 1.34 ; 
Table 1 Baseline participant characteristics

\begin{tabular}{|c|c|c|c|c|}
\hline & \multicolumn{2}{|l|}{ Diabetes $(n=163)$} & \multicolumn{2}{|c|}{ Hypertension $(n=105)$} \\
\hline & Free distribution & Usual access & Free distribution & Usual access \\
\hline & Number (\%) & Number (\%) & Number (\%) & Number (\%) \\
\hline & $(n=83)$ & $(n=80)$ & $(n=56)$ & $(n=49)$ \\
\hline Women & $35(42.2)$ & $35(43.8)$ & $22(39.3)$ & $17(34.7)$ \\
\hline Age (mean, SD) & $59 \pm 10$ & $58 \pm 11.2$ & $60 \pm 8.2$ & $61 \pm 9.3$ \\
\hline Age 65 years or older & $25(30.1)$ & $19(23.8)$ & $17(30.4)$ & $16(32.7)$ \\
\hline \multicolumn{5}{|l|}{ Ethnicity } \\
\hline White & $42(50.6)$ & $53(66.3)$ & $46(82.1)$ & $34(69.4)$ \\
\hline Black & $9(10.8)$ & $10(12.5)$ & $2(3.6)$ & $4(8.1)$ \\
\hline $\begin{array}{l}\text { Southeast or East Asian (incl Korean, Japanese, } \\
\text { Filipino, Chinese) }\end{array}$ & $6(7.2)$ & $2(2.5)$ & $4(7.1)$ & $2(4.1)$ \\
\hline South Asian & $14(16.9)$ & $9(11.3)$ & $1(1.8)$ & $3(6.1)$ \\
\hline Latin American & $1(1.2)$ & $3(3.8)$ & $0(0.0)$ & $2(4.1)$ \\
\hline West Asian (including Arab) & $2(2.4)$ & $1(1.3)$ & $0(0.0)$ & $0(0.0)$ \\
\hline Mixed or other & $9(10.8)$ & $2(2.5)$ & $2(3.6)$ & $4(8.2)$ \\
\hline Declined to provide & $0(0.0)$ & $0(0.0)$ & $4(7.1)$ & $0(0.0)$ \\
\hline \multicolumn{5}{|l|}{ Main income source } \\
\hline Wages and salaries (including self-employed) & $44(53.0)$ & $38(47.5)$ & $30(53.6)$ & $28(57.1)$ \\
\hline Pension & $22(26.5)$ & $19(23.8)$ & $14(25.0)$ & $9(18.4)$ \\
\hline Social support (eg, welfare or disability) & $11(13.3)$ & $13(16.3)$ & $4(7.1)$ & $8(16.3)$ \\
\hline Unemployment insurance & $4(4.8)$ & $3(3.8)$ & $4(7.1)$ & $2(4.1)$ \\
\hline Other & $0(0.0)$ & $1(1.3)$ & $0(0.0)$ & $0(0.0)$ \\
\hline Declined to provide & $2(2.4)$ & $6(7.5)$ & $4(7.1)$ & $2(4.1)$ \\
\hline \multicolumn{5}{|l|}{ Household income } \\
\hline$\$ C 30000$ or less & $46(55.4)$ & $41(51.3)$ & $23(41.1)$ & $19(38.8)$ \\
\hline$\$ C 30000-\$ C 70000$ & $24(28.9)$ & $22(27.5)$ & $12(21.4)$ & $12(24.5)$ \\
\hline$\$ C 70000$ or greater & $3(3.6)$ & $4(5.0)$ & $4(7.1)$ & $0(0.0)$ \\
\hline Declined to provide & $10(12.0)$ & $13(16.3)$ & $17(30.4)$ & $18(36.7)$ \\
\hline Number of medicines prescribed at baseline (mean, SD) & $5 \pm 2.8$ & $5 \pm 3.1$ & $4 \pm 2.0$ & $4 \pm 2.6$ \\
\hline Urban site & $50(60.2)$ & $48(60.0)$ & $22(39.3)$ & $27(55.1)$ \\
\hline Rural site & $33(39.8)$ & $32(40.0)$ & $34(60.7)$ & $22(44.9)$ \\
\hline
\end{tabular}

$\mathrm{p}=0.44$ ) for patients receiving free distribution compared with those with usual medicine access. There was a small increase in SMBG (aRR 1.30; 95\% CI 0.66 to 2.57; $\mathrm{p}=0.45$ ) (see table 2). There were no differences in appointments with primary care providers or consultants, but there was a trend toward fewer missed appointments with primary care providers (aRR $0.80 ; 95 \%$ CI 0.48 to $1.33 ; \mathrm{p}=0.39$ ) (see table 2). There was no difference between the free distribution and usual access groups with respect to net change in medicine prescriptions. Overall, the net change in the number of medicines prescribed to participants receiving free distribution was 14 new starts (a total of 19 new medicines started and 5 medicines stopped; average of 0.17 new medicines per person) and the net change for those with usual access was 14 new starts (a total of 21 new medicines started and 7 medicines stopped; average of 0.18 new medicines per person).
Impact of free distribution of medicines in subgroup of people with hypertension

Among patients with hypertension, free distribution was associated with a lower rate of serum Cr (aRR 0.61; $95 \%$ CI 0.38 to $0.97 ; \mathrm{p}=0.04$ ) and electrolyte measuring (aRR $0.59 ; 95 \%$ CI 0.36 to $0.98 ; \mathrm{p}=0.04)$, and fewer missed appointments (aRR $0.41 ; 95 \%$ CI 0.18 to $0.90 ; \mathrm{p}=0.03$ ) (see table 3 ). There were trends towards fewer encounters with primary care providers (aRR 0.90; 95\% CI 0.71 to $1.10 ; \mathrm{p}=0.25$ ) and consultants (aRR $0.59 ; 95 \%$ CI 0.07 to $4.62 ; \mathrm{p}=0.61$ ) but similar self-monitoring of blood pressure (aOR $1.10 ; 95 \%$ CI 0.38 to 3.17 ; $\mathrm{p}=0.86$ ) (see table 3 ). There was no difference in blood pressure measuring in clinic. There were slightly more new medicine starts in participants receiving free distribution. Overall, the net change in the number of medicines prescribed to intervention participants was 15 new starts (a total of 20 new 
Table 2 Diabetes process of care indicators

\begin{tabular}{|c|c|c|c|c|}
\hline & $\begin{array}{l}\text { Free medicine } \\
\text { distribution }\end{array}$ & $\begin{array}{l}\text { Usual medicine } \\
\text { access }\end{array}$ & $\begin{array}{l}\text { Unadjusted } \\
\text { difference }\end{array}$ & Adjusted difference \\
\hline HbA1c measurements & $2(2)(187)$ & $2(2)(160)$ & $\begin{array}{l}1.13(0.91 \text { to } 1.39) \\
p=0.27\end{array}$ & $\begin{array}{l}1.09(0.88 \text { to } 1.34) \\
p=0.44\end{array}$ \\
\hline BP measurements & $3(7)(278)$ & $3(7)(274)$ & $\begin{array}{l}0.98(0.78 \text { to } 1.23) \\
p=0.85\end{array}$ & $\begin{array}{l}0.96(0.77 \text { to } 1.19) \\
\mathrm{p}=0.71\end{array}$ \\
\hline LDL-c measurements & $1(1)(65)$ & $1(1)(61)$ & $\begin{array}{l}1.03(0.72 \text { to } 1.46) \\
p=0.88\end{array}$ & $\begin{array}{l}0.99(0.70 \text { to } 1.41) \\
p=0.96\end{array}$ \\
\hline Urine ACR measured & $54 \%(45 / 83)$ & $58 \%(46 / 80)$ & $\begin{array}{l}0.88 \text { (0.47 to } 1.63) \\
\mathrm{p}=0.67\end{array}$ & $\begin{array}{l}0.88 \text { (0.47 to } 1.67) \\
\mathrm{p}=0.70\end{array}$ \\
\hline Serum creatinine measured & $82 \%(68 / 83)$ & $76 \%(61 / 80)$ & $\begin{array}{l}1.41 \text { (0.66 to } 3.02) \\
p=0.37\end{array}$ & $\begin{array}{l}1.33(0.61 \text { to } 2.91) \\
p=0.48\end{array}$ \\
\hline Foot examination performed & $63 \%(52 / 83)$ & $61 \%(49 / 80)$ & $\begin{array}{l}1.06 \text { (0.56 to } 2.00) \\
p=0.85\end{array}$ & $\begin{array}{l}0.94 \text { (0.49 to } 1.84) \\
\mathrm{p}=0.87\end{array}$ \\
\hline Eye examination performed & $42 \%(35 / 83)$ & $43 \%(34 / 80)$ & $\begin{array}{l}0.99(0.53 \text { to } 1.84) \\
p=0.97\end{array}$ & $\begin{array}{l}1.03(0.53 \text { to } 2.01) \\
p=0.93\end{array}$ \\
\hline Influenza vaccine administered & $29 \%(24 / 83)$ & $28 \%(22 / 80)$ & $\begin{array}{l}1.07(0.54 \text { to } 2.12) \\
\mathrm{p}=0.84\end{array}$ & $\begin{array}{l}1.08(0.52 \text { to } 2.22) \\
\mathrm{p}=0.84\end{array}$ \\
\hline Self-monitoring of blood glucose & $54 \%(45 / 83)$ & $48 \%(38 / 80)$ & $\begin{array}{l}1.31 \text { (0.71 to } 2.42) \\
p=0.39\end{array}$ & $\begin{array}{l}1.30(0.66 \text { to } 2.57) \\
p=0.45\end{array}$ \\
\hline Primary care encounters related to diabetes & $3(6)(258)$ & $3(5)(243)$ & $\begin{array}{l}1.02(0.81 \text { to } 1.30) \\
p=0.85\end{array}$ & $\begin{array}{l}1.02(0.81 \text { to } 1.28) \\
\mathrm{p}=0.90\end{array}$ \\
\hline Consultant encounters related to diabetes & $1(1)(49)$ & $1(1)(51)$ & $\begin{array}{l}0.93 \text { (0.53 to } 1.62) \\
p=0.79\end{array}$ & $\begin{array}{l}1.01(0.59 \text { to } 1.75) \\
p=0.96\end{array}$ \\
\hline Missed primary care appointments & $1(1)(43)$ & $1(1)(49)$ & $\begin{array}{l}0.85 \text { (0.50 to } 1.44) \\
p=0.54\end{array}$ & $\begin{array}{l}0.80 \text { (0.48 to } 1.33) \\
p=0.39\end{array}$ \\
\hline $\begin{array}{l}\text { Total number of encounters and manoeuvres } \\
\text { (assign } 0 \text { or } 1 \text { for binary indicators; exclude } \\
\text { missed appointments) }\end{array}$ & $13(42)(1106)$ & $13(38)(1039)$ & $\begin{array}{l}1.03(0.88 \text { to } 1.19) \\
\mathrm{p}=0.74\end{array}$ & $\begin{array}{l}1.01(0.88 \text { to } 1.17) \\
p=0.85\end{array}$ \\
\hline
\end{tabular}

Count indicators are reported as the mean number of measurements or encounters with the variance and the sum of all measurements or encounters. Binary indicators are reported as a proportion. Differences are adjusted for age, sex and site, and reported as rate ratio or OR with the $95 \% \mathrm{Cl}$ and $\mathrm{p}$ value.

ACR, albumin to creatinine ratio; BP, blood pressure; HbA1c, glycated haemoglobin; LDL-c, low-density lipoprotein-cholesterol.

medicines started and 5 medicines stopped; average: 0.27 new medicines per person) and the net change for control participants was 0 (a total of nine new medicines started and nine medicines stopped).

\section{DISCUSSION}

In this post hoc analysis of randomised controlled trial findings, free distribution of medicines to people with diabetes or hypertension was not associated with more visits to primary care providers or consultants and, in fact, patients with hypertension had less laboratory monitoring and slightly fewer visits. Free distribution may slightly increase self-monitoring and reduce missed appointments.

The modest reductions in laboratory testing of serum $\mathrm{Cr}$ and electrolytes for patients with hypertension may reflect appropriate clinical judgement against repeat testing. The Canadian guidelines recommend that the frequency of laboratory testing should be guided by clinical judgement and no specific intervals are mentioned in the guidelines. Clinicians may have been less likely to order laboratory testing in patients receiving free distribution because they had slightly better control of their blood pressure, possibly due to the greater number of medicines prescribed. These tests may also have been ordered less frequently because patients had fewer visits, potentially because they were self-monitoring. Systematic reviews have reported improved glycaemic control in patients with diabetes performing SMBG, and reduced blood pressure in patients with hypertension selfmeasuring their blood pressure. ${ }^{15} 16$ Thus, the observed trend towards more self-monitoring, if real, could reflect 
Table 3 Hypertension process of care indicators

\begin{tabular}{|c|c|c|c|c|}
\hline & $\begin{array}{l}\text { Free medicine } \\
\text { distribution }\end{array}$ & $\begin{array}{l}\text { Usual medicine } \\
\text { access }\end{array}$ & $\begin{array}{l}\text { Unadjusted } \\
\text { difference }\end{array}$ & $\begin{array}{l}\text { Adjusted } \\
\text { difference }\end{array}$ \\
\hline BP measurements & $3(4)(173)$ & $3(5)(160)$ & $\begin{array}{l}0.95(0.74 \text { to } 1.22) \\
p=0.67\end{array}$ & $\begin{array}{l}0.99(0.77 \text { to } 1.27) \\
p=0.92\end{array}$ \\
\hline HbA1c measurements & $1(1)(37)$ & $1(1)(42)$ & $\begin{array}{l}0.77(0.49 \text { to } 1.22) \\
p=0.27\end{array}$ & $\begin{array}{l}0.83(0.53 \text { to } 1.30) \\
p=0.41\end{array}$ \\
\hline Lipid measurements & $1(1)(36)$ & $1(1)(37)$ & $\begin{array}{l}0.85 \text { ( } 0.54 \text { to } 1.35) \\
p=0.49\end{array}$ & $\begin{array}{l}0.91(0.57 \text { to } 1.46) \\
p=0.70\end{array}$ \\
\hline Serum creatinine measurements & $1(4)(78)$ & $2(13)(110)$ & $\begin{array}{l}0.62(0.39 \text { to } 1.00) \\
p=0.05\end{array}$ & $\begin{array}{l}0.61 \text { ( } 0.38 \text { to } 0.97) \\
p=0.04\end{array}$ \\
\hline Serum electrolyte measurements & $1(3)(66)$ & $2(11)(103)$ & $\begin{array}{l}0.56(0.34 \text { to } 0.93) \\
p=0.02\end{array}$ & $\begin{array}{l}0.59(0.36 \text { to } 0.98) \\
p=0.04\end{array}$ \\
\hline Primary care encounters & $5(9)(287)$ & $6(14)(302)$ & $\begin{array}{l}0.83(0.66 \text { to } 1.05) \\
p=0.11\end{array}$ & $\begin{array}{l}0.9(0.71 \text { to } 1.10) \\
p=0.25\end{array}$ \\
\hline Consultant encounters related to hypertension & $0(0)(5)$ & $0(0)(5)$ & $\begin{array}{l}0.88(0.13 \text { to } 6.10) \\
p=0.89\end{array}$ & $\begin{array}{l}0.59(0.07 \text { to } 4.62) \\
p=0.61\end{array}$ \\
\hline Missed primary care appointments & $0(0)(14)$ & $0(4)(44)$ & $\begin{array}{l}0.28(0.12 \text { to } 0.64) \\
p=0.00\end{array}$ & $\begin{array}{l}0.41(0.18 \text { to } 0.90) \\
p=0.03\end{array}$ \\
\hline Self-monitoring of BP & $21 \%(12 / 56)$ & $18 \%(9 / 49)$ & $\begin{array}{l}1.21(0.46 \text { to } 3.18) \\
p=0.70\end{array}$ & $\begin{array}{l}1.10(0.38 \text { to } 3.17) \\
p=0.86\end{array}$ \\
\hline $\begin{array}{l}\text { Total number of encounters and manoeuvres } \\
\text { (assign } 0 \text { or } 1 \text { for binary indicators; exclude } \\
\text { missed appointments) }\end{array}$ & $12(43)(694)$ & $16(97)(768)$ & $\begin{array}{l}0.79(0.63 \text { to } 1.00) \\
p=0.04\end{array}$ & $\begin{array}{l}0.83(0.67 \text { to } 1.04) \\
p=0.10\end{array}$ \\
\hline
\end{tabular}

Count indicators are reported as the mean number of measurements or encounters with the variance and the sum of all measurements or encounters. Binary indicators are reported as a proportion. Differences are adjusted for age, sex and site, and reported as OR or rate ratio with the $95 \% \mathrm{Cl}$ and $\mathrm{p}$ value.

BP, blood pressure; HbA1c, glycated haemoglobin.

improved patient motivation, better disease control or different guidance from clinicians. A 2018 randomised controlled trial found that using self-monitored blood pressure readings to titrate antihypertensive treatments led to a significant reduction in blood pressure compared with the use of clinic readings to guide care ${ }^{17}$ In this trial, patients with hypertension had a lower systolic blood pressure after 1 year. The improvements in disease control and usefulness of self-measured blood pressure readings may have resulted in clinicians asking patients to monitor their blood pressure at home rather than attend clinic; this would explain both the increase in self-monitoring and the reduction in clinic visits. A 1985 controlled trial of the effects of medical insurance on health spending and health status reported lower blood pressure with free care, though the cause of the difference was additional contact with physicians under free care. ${ }^{18}$

The reduction in missed appointments observed here may be explained by an improved clinician-patient relationship and better perceived disease control. The reduction in missed appointments did not relate to needing to attend appointments in order to get their free medications, as the study pharmacist had access to their electronic medical record, could communicate with primary care providers, and medications were mailed to participants. A 2004 study of patient perceptions found that emotional barriers (including the fear of bad news) and perceived disrespect by the healthcare system caused patients to miss primary care appointments. ${ }^{19}$ Additionally, a 2014 cross-sectional survey reported that patients with hypertension with no medication coverage and high medication costs were more likely to miss appointments. ${ }^{20}$ Patients may not take their medicines due to cost and may miss appointments due to feelings of embarrassment or guilt over this; this may be obviated by free distribution of medicines.

Our study found that there was only a small nonsignificant increase in HbA1c monitoring and serum $\mathrm{Cr}$ monitoring in patients with diabetes. Our findings suggest that financial barriers to medication access may not deter patients with diabetes from engaging in necessary health visits and screening related to the management of their condition. In contrast, a study of American patients with diabetes found that lower cost-related non-adherence was associated with improved compliance to annual diabetes recommendations. ${ }^{5}$ Financial incentives to clinicians, audit and feedback interventions, and reminders to clinicians can achieve modest reductions in HbAlc, and our 
study found a small increase in the frequency of HbA1c monitoring with free medicine distribution. ${ }^{6}$

The results of this study post hoc analysis of trial findings suggest that improving access to chronic disease medicines will not substantially increase costs associated with outpatient visits. To the contrary, in this study free distribution appeared to increase self-monitoring, reduce visits for hypertension and reduce the total number of healthcare encounters and manoeuvres performed in a year, without changing the likelihood of visits for diabetes. Increasing access to medicines may encourage self-monitoring practices, reduce in-person visits and decrease laboratory investigations performed. Free distribution of medicines may not only improve blood pressure control but could also reduce the per-person costs associated with the management of hypertension.

Strengths of this study include the fact that the results are based on a randomised controlled trial. Participants differed with respect to income level, ethnicity and location (urban vs rural). Despite the fact that this was a post hoc analysis and randomisation was not stratified based on these characteristics, we found that the groups were largely balanced; except for urban status. There are also some limitations in this analysis. Associations identified during post hoc analyses could be spurious and thus the findings should be viewed as hypothesis-generating. ${ }^{21}$ The study population is a subset of the CLEAN Meds trial, and only included those with diabetes or hypertension based on whether they were prescribed at least one diabetic or antihypertensive agent at the start of the trial; this reduced sample size is a limitation. The trial was not designed to have sufficient power to detect differences in some of the outcomes examined in this study so the failure to identify associations should be interpreted with caution. We separated participants with diabetes from those with hypertension while we could have analysed some shared outcomes (eg, blood pressure measurement) using a single group with a larger sample size. Since the trial was unblinded, patients and clinicians could have been motivated by allocation to free access to improve the process of care. The trial was conducted with primary care patients in a high-income country who reported cost-related nonadherence and the findings may not apply in other settings. The study was based on a review of primary care charts that do not reflect every actual encounter (eg, visits to other providers).

\section{CONCLUSION}

This post hoc analysis of randomised controlled trial results found that free distribution of medicines may improve self-monitoring behaviours and reduce missed primary care appointments for patients with diabetes or hypertension. Free distribution may also reduce primary care and consultant appointments and laboratory testing in patients with hypertension. Additionally, free distribution of medicines improves disease control and improves patients' self-reported care. ${ }^{10}$ Overall, these findings suggest that improving medicine accessibility for patients with diabetes and hypertension not only improves surrogate health outcomes but also improves the patient experience and may also reduce healthcare costs by encouraging self-monitoring practices. The hypotheses generated by this post hoc analysis of randomised controlled trial findings could be tested in future studies.

\section{Author affiliations}

${ }^{1}$ Faculty of Medicine, University of Toronto, Toronto, Ontario, Canada

${ }^{2}$ MAP Centre for Urban Health Solutions, St Michael's Hospital, Toronto, Ontario, Canada

${ }^{3}$ Department of Medicine, Cumming School of Medicine, University of Calgary, Calgary, Alberta, Canada

${ }^{4}$ Department of Community Health Sciences, Cumming School of Medicine, University of Calgary, Calgary, Alberta, Canada

${ }^{5} 0$ 'Brien Institute for Public Health, Cumming School of Medicine, University of Calgary, Calgary, Alberta, Canada

${ }^{6}$ Libin Cardiovascular Institute, Cumming School of Medicine, University of Calgary, Calgary, Alberta, Canada

${ }^{7}$ Institute for Clinical Evaluative Sciences, Toronto, Ontario, Canada

${ }^{8}$ Department of Family and Community Medicine, University of Toronto, Toronto, Ontario, Canada

${ }^{9}$ Institute of Health Policy, Management, and Evaluation, University of Toronto, Toronto, Ontario, Canada

${ }^{10}$ Department of Medicine, Sunnybrook Health Sciences Centre, Toronto, Ontario, Canada

${ }^{11}$ Department of Family and Community Medicine, St.Michael's Hospital, Toronto, Ontario, Canada

Contributors $\mathrm{OC}$ contributed to the data curation, formal analysis, investigation, methodology, visualisation, writing the original draft, and reviewing and editing. HW contributed to the data curation, formal analysis, investigation, writing the original draft, and reviewing and editing. MA contributed to the data curation, formal analysis, and reviewing and editing. BM contributed to the methodology, validation, investigation, resources, and reviewing and editing. BRS contributed to the methodology, validation, investigation, resources, and reviewing and editing. RW contributed to methodology, formal analysis, investigation, resources, and reviewing and editing. NP contributed to the conceptualisation, methodology, validation, formal analysis, investigation, resources, writing the original draft, and reviewing and editing.

Funding This work was supported by the Canadian Institutes of Health Research (grant number 381409) and the Keenan Research Summer Student Program (award number 2019).

Disclaimer They played no role in study design; in the collection, analysis and interpretation of data; in the writing of the report and in the decision to submit the article for publication.

Competing interests NP reports grants from the Canadian Institutes for Health Research, the Ontario SPOR Support Unit, the Canada Research Chairs programme and Physicians Services Incorporated during the conduct of the study. All other authors (OC, HW, MA, BM and BRS) declare that they have no competing interests.

Patient and public involvement Patients and/or the public were not involved in the design, or conduct, or reporting, or dissemination plans of this research.

Patient consent for publication Not required.

Provenance and peer review Not commissioned; externally peer reviewed.

Data availability statement Data are available upon reasonable request. Deidentified participant data are available upon reasonable request from the corresponding author.

Open access This is an open access article distributed in accordance with the Creative Commons Attribution Non Commercial (CC BY-NC 4.0) license, which permits others to distribute, remix, adapt, build upon this work non-commercially, and license their derivative works on different terms, provided the original work is properly cited, appropriate credit is given, any changes made indicated, and the use is non-commercial. See: http://creativecommons.org/licenses/by-nc/4.0/.

ORCID iD

Nav Persaud http://orcid.org/0000-0003-3327-5580 


\section{REFERENCES}

1 Braga MFB, Casanova A, Teoh $\mathrm{H}$, et al. Treatment gaps in the management of cardiovascular risk factors in patients with type 2 diabetes in Canada. Can J Cardiol 2010;26:297-302.

2 Saaddine JB, Engelgau MM, Beckles GL, et al. A diabetes report card for the United States: quality of care in the 1990s. Ann Intern Med 2002;136:565.

3 Hajjar I, Kotchen TA. Trends in prevalence, awareness, treatment, and control of hypertension in the United States, 1988-2000. JAMA 2003;290:199-206.

4 Law MR, Cheng L, Dhalla IA, et al. The effect of cost on adherence to prescription medications in Canada. Can Med Assoc $J$ 2012;184:297-302.

5 Kang H, Lobo JM, Kim S, et al. Cost-related medication nonadherence among U.S. adults with diabetes. Diabetes Res Clin Pract 2018;143:24-33.

6 Tricco AC, Ivers NM, Grimshaw JM, et al. Effectiveness of quality improvement strategies on the management of diabetes: a systematic review and meta-analysis. Lancet 2012;379:2252-61.

7 Walsh JME, McDonald KM, Shojania KG, et al. Quality improvement strategies for hypertension management: a systematic review. Med Care 2006;44:646-57.

8 Rosella LC, Lebenbaum M, Fitzpatrick T, et al. Impact of diabetes on healthcare costs in a population-based cohort: a cost analysis. Diabetic Medicine 2016;33:395-403.

9 Weaver CG, Clement FM, Campbell NRC, et al. Healthcare costs attributable to hypertension: Canadian population-based cohort study. Hypertension 2015;66:502-8.

10 Persaud N, Bedard M, Boozary AS, et al. Effect on treatment adherence of distributing essential medicines at no charge: the clean Meds randomized clinical trial. JAMA Intern Med 2019
11 Murray CM, Shah BR. Diabetes self-management education improves medication utilization and retinopathy screening in the elderly. Prim Care Diabetes 2016;10:179-85.

12 An J, JaeJin A. The impact of patient-centered medical homes on quality of care and medication adherence in patients with diabetes mellitus. J Manag Care Spec Pharm 2016;22:1272-84.

13 Diabetes Canada. Clinical practice guidelines - 2018 full guidelines [online]. Available: http://guidelines.diabetes.ca/cpg [Accessed 23 Sep 2019].

14 Diagnosis \& Assessment | Hypertension Canada Guidelines [online]. Available: https://guidelines.hypertension.ca/diagnosis-assessment/ [Accessed 23 Sep 2019].

15 Zhu H, Zhu Y, Leung S-W. Is self-monitoring of blood glucose effective in improving glycaemic control in type 2 diabetes without insulin treatment: a meta-analysis of randomised controlled trials. BMJ Open 2016;6:e010524.

16 Uhlig K, Patel K, Ip S, et al. Self-measured blood pressure monitoring in the management of hypertension: a systematic review and metaanalysis. Ann Intern Med 2013;159:185-94.

17 McManus RJ, Mant J, Franssen M, et al. Efficacy of self-monitored blood pressure, with or without telemonitoring, for titration of antihypertensive medication (TASMINH4): an unmasked randomised controlled trial. Lancet 2018;391:949-59.

18 Keeler EB, Brook RH, Goldberg GA, et al. How free care reduced hypertension in the health insurance experiment. JAMA 1985;254:1926-31

19 Lacy NL, Paulman A, Reuter MD, et al. Why we don't come: patient perceptions on no-shows. Ann Fam Med 2004;2:541-5.

20 Nwabuo CC, Dy SM, Weeks K, et al. Factors associated with appointment non-adherence among African-Americans with severe, poorly controlled hypertension. PLoS One 2014;9:e103090.

21 Pocock SJ, Assmann SE, Enos LE, et al. Subgroup analysis, covariate adjustment and baseline comparisons in clinical trial reporting: current practiceand problems. Stat Med 2002;21:2917-30. 